Article

\title{
Determination of Wide Burnishing Energy-Force Parameters based on Constructing the Kinematically Admissible Velocity Field
}

\author{
Igor Bobrovskij ${ }^{1, *}$, Alexander Khaimovich ${ }^{2}$, Nikolaj Bobrovskij ${ }^{3}$ and Aleksander D'yakonov ${ }^{4}$ \\ 1 Samara Scientific Center of Russian Academy of Science, Samara 443001, Russia \\ 2 Department of Engine Production Technology, Samara National Research University, Samara 443086, Russia; \\ berill_samara@bk.ru \\ 3 Equipment and technology of machine-building production, Togliatti State University, Togliatti 445020, \\ Russia; bobrnm@yandex.ru \\ 4 Technology of automated mechanical engineering, South Ural State University, Chelyabinsk 454080, Russia; \\ sigma-80@mail.ru \\ * Correspondence: bobri@yandex.ru
}

Received: 5 November 2019; Accepted: 23 December 2019; Published: 25 December 2019

\begin{abstract}
Processing technology using productive surface plastic deformation (SPD) was developed and is known as "wide burnishing" (WB). The mechanics of new WB technology differs from classic SPD technologies (rolling or burnishing). For example, force, applied during burnishing, is equal to $150-300 \mathrm{~N}$, but for $\mathrm{WB}$ it is equal to $2500-5000 \mathrm{~N}$ due to implementing this process in mass production, characterized with limited processing time (3-4 turns of the work piece). WB also has a high degree of deformation due to a multiple deformation passes. An analytical study of burnishing with the upper bound approach method energy-force parameters was carried out. Its results were compared with the results of finite elements analysis in Deform 2D and with experimental data. Field of reasonable burnishing parameters, assuring minimum surface roughness, was determined experimentally.
\end{abstract}

Keywords: surface plastic deformation; wide burnishing; indenter pressure; upper bound approach method; FEM

\section{Introduction}

Surface plastic deformation by rigid tools is used in engineering technology to increase wear and fatigue resistance of metal contact parts. Roller burnishing is a cost effective surface enhancement process where a ceramic ball rolls on the machined surface under a high pressure and flattens the roughness peaks. It not only improves surface finish but also imposes favorable compressive residual stresses and raises hardness in functional surfaces, which can lead to long fatigue life [1]. Recently research in roller burnishing was based on experimental studies. Experimental data have been reported worldwide by many researchers [2-6]. Roettger [7] developed a 2D FEM model for roller burnishing using commercial FEM software DEFORM-2D 3D. The model from [7] has been further improved by Yen et al. [1] using DEFORM-2D and DEFORM-3D. The simulation results (i.e., surface deformation and residual stress) were evaluated and compared between initial hard turned and burnished surfaces. In recent studies, FE analysis of rigid cylinder rolling along elastic-plastic half space was considered $[8,9]$. Steady state ideal plastic flow induced by rolling of rigid cylinder is considered in [10] using a small parameter approach. Approximate analysis related with the rolling friction is given in [11]. Nepershin [12-14] developed a steady state plastic flow model of surface layer for rigid circular and elliptical cylinder sliding with contact friction consideration. 
Wide burnishing (Figure 1) radically differs from classical burnishing by interaction conditions (processing is performed without line feed, but only with cross-feed; beside this, the tool diameter is equal to the work surface length) and processing conditions (classic burnishing force is equal to $150 \mathrm{~N}$, for wide burnishing it can reach up to $5000 \mathrm{~N}$ ).

This work is aimed at evaluating the plastic deformation mechanism in wide burnishing by theoretical and experimental study of process parameters influence on processed surface texture.

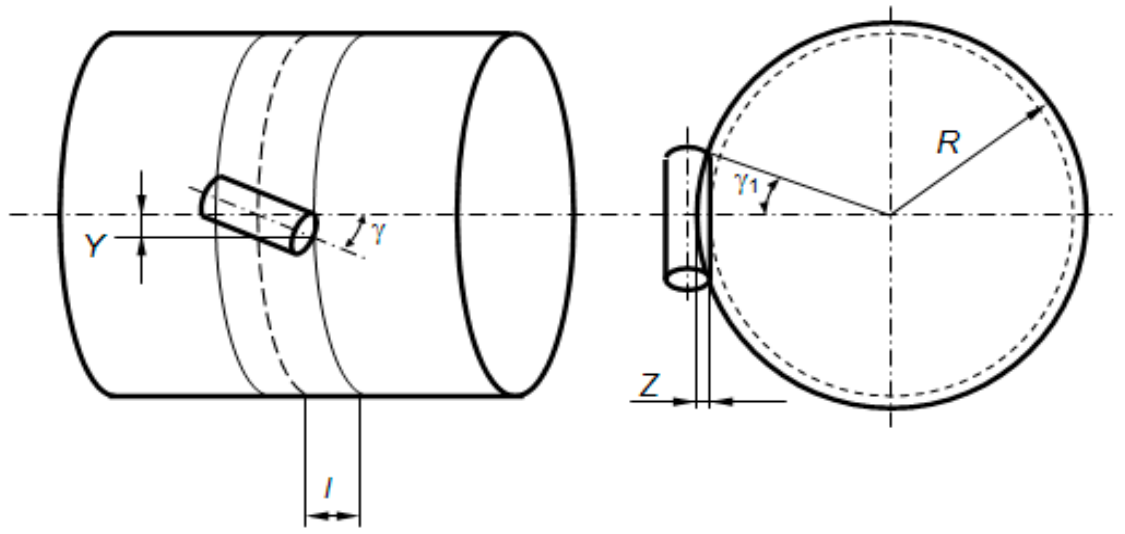

Figure 1. Wide burnishing geometrical parameters.

Wide burnishing machine (Figure 2) includes a closed hydraulic system for tools operating. The device consists of rod (2) placed in the casing inner cavity (3) and is equipped with a tool (1). Manometer (4) is connected to the casing inner cavity, filled with liquid. Screw (6) is fitted with a piston and a flywheel and regulates pressure in the casing cavity. Screw (5) provides circumferential and axial clamping of rod (2). Manometer elastic element of this device acts as a damper. Pressure changing in the hydraulic system provides the required burnishing force. General view of the device is shown in Figure 2.

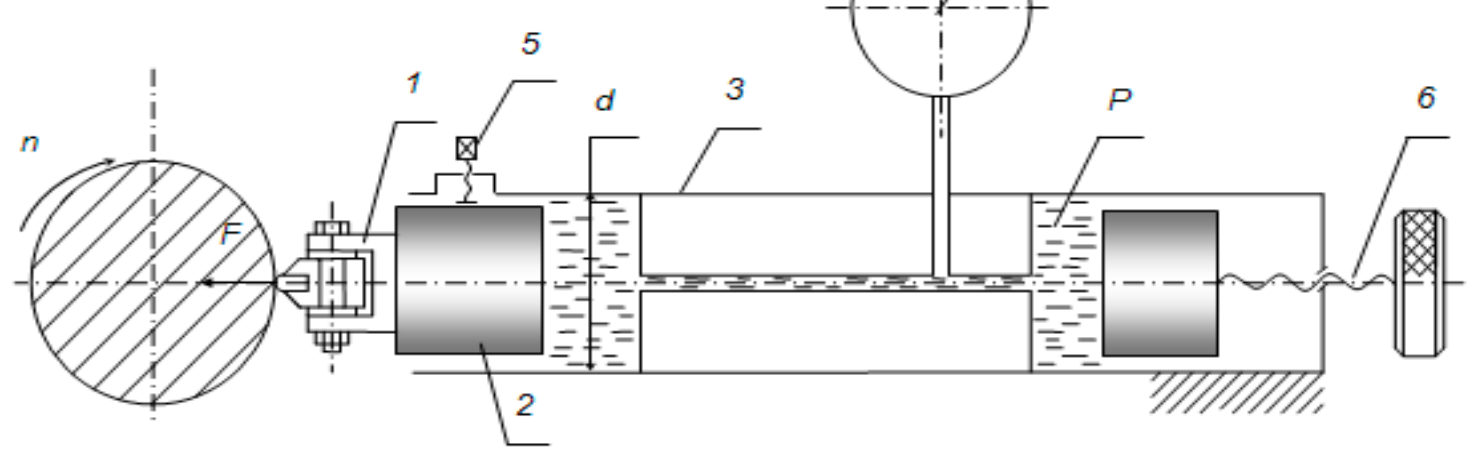

Figure 2. General view of the WB device.

The present research is aimed at analytic determining the wide burnishing (WB) energy-force parameters, such as indenter pressing force and average deformation rate at the plastic deformation site when moving indenter penetrates the work piece surface.

\section{Theoretical Background}

In the present study the upper bound method is used to develop a steady state plastic flow model of WB. Upper bound method is based on the deformation energy calculations. This method needs 
velocity field calculation. The power of deformation is calculated from the velocity field, which is determined using known information regarding the metal flow. This approach is presently widely substituted by the FEM. There are, however, some advantages of this method, which allow formulation of efficient optimization tasks for industrial processes [15]. Upper bound approach was first formulated by Prager and Hodge [16] and is based on following assumptions:

(a) Among all kinematically admissible velocity fields in the deformation zone, there is only one which causes the total power calculated as a product of stress and strain reaches minimum. This total power is the sum of the power of plastic deformation, power at tool-workpiece interface, and the power dissipated at the velocity discontinuity surfaces.

(b) Strain rate field calculated from kinematically admissible velocity field is also kinematically admissible.

(c) Real power of deformation supplied to the system by the external forces is always lower than power calculated as sum of powers by first item. If the velocity field is determined with an error, the power calculated from this field will be larger than the real power of the process. The minimum of the power is obtained for the real (correct) velocity field.

The plastic metal processing problems solution is based on a kinematically admissible velocity field, used to construct deformation rate fields with further transition to energy-force parameters [17-19]. It is much simpler than solutions based on admissible stress fields, satisfying boundary conditions and equilibrium criterion. Deformation rate fields are easily constructed even under dynamic loading conditions [20]. A solution of the contact problem for burnishing, involving conformal mapping, was described in [21]. The theory presented below is based on the upper bound approach for evaluating the energy-force burnishing parameters stemming from the kinematically admissible velocity field with tangent component discontinuities [22].

Assumptions made to WB with upper bound approach:

(a) Work piece of rigid-plastic material without strain hardening;

(b) Task is solved in plain conditions (plain-deformed state);

(c) Plastic deformation site is a second-order curve bounded by the indenter surface

(d) Microroughness is neglected; the indenter penetrates flat surface at depth $t$ (Figure 3).

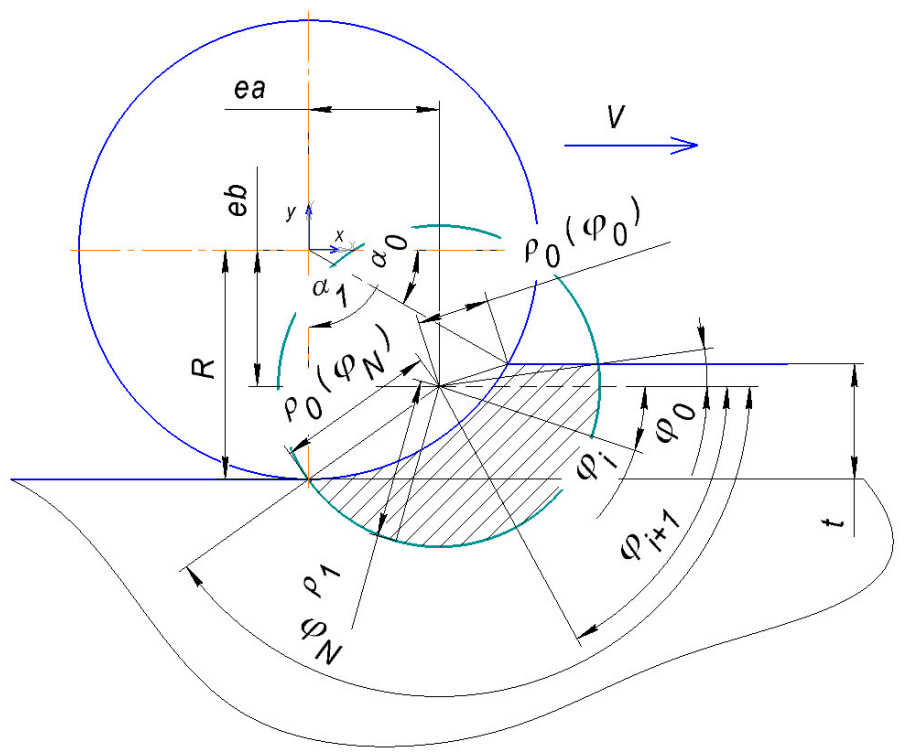

Figure 3. Forming velocity field during wide burnishing.

Suppose a cylinder-shaped indenter with radius $R$ penetrates a half-plane at depth of $t$. We construct kinematically admissible velocity field in the cylindrical coordinate system $\rho, \varphi, z$ with 
its center located at the distance $e a, e b$ from the indenter axis. Z-axis is aligned with the indenter axis, indenter generator line $L>>R_{0}$, which allows assuming plane-deformed state $\left(\varepsilon_{z z}=0\right)$. Suppose $\rho_{1}$ is the radius-vector of a circle with center $O(0,0,0)$. Let us take velocity of material, displaced while burnishing, at circumference of radius $\rho_{1}$ equal to zero:

$$
v_{\rho}\left(\rho_{1}\right)=0
$$

The boundary of continuous medium in the contact with the indenter zone (arc of a circle of radius $R$ ) moves with the indenter linear velocity $V$. Supposing the displaced material flows only along the $\rho$-axis (assumption 1), the following boundary condition is satisfied:

$$
v_{\rho}\left(\rho_{0}(\varphi)\right) \cdot \cos \varphi=V
$$

where $\rho_{0}(\varphi)$-arc of the indenter and burnished material contact area in coordinates $\rho, \varphi$.

Variables var $=\left(e a, e b, \rho_{1}\right)$ under boundary conditions Equations (1) and (2) determine the kinematically admissible velocity field.

Deformation rate tensor components in a cylindrical coordinates system have following form:

$$
\dot{\varepsilon}_{\rho \rho}=\frac{\partial v_{\rho}}{\partial \rho} ; \dot{\varepsilon}_{\varphi \varphi}=\frac{1}{\rho} \frac{\partial v_{\varphi}}{\partial \varphi}+\frac{v_{\rho}}{\rho}
$$

Since according to the accepted assumption the displaced material velocity in the plastic deformation site, changes only along the radius $\rho$ i.e.,

$$
\frac{\partial v_{\rho}}{\partial \varphi}=0 ; \frac{\partial v_{\varphi}}{\partial \varphi}=0
$$

we can substitute Equation (4) and values of strain rates Equation (3) in the incompressibility condition (5).

$$
\dot{\varepsilon}_{\rho \rho}+\dot{\varepsilon}_{\varphi \varphi}=0
$$

and derive the following Equation (6).

$$
\frac{\partial v_{\rho}}{\partial \rho}+\frac{v_{\rho}}{\rho}=0
$$

The solution of differential equation (6) can be presented in the form Equation (7).

$$
v_{\rho}=C_{v}\left(\frac{1}{\rho}-\frac{1}{\rho_{1}}\right)
$$

where constant $C_{v}$ is determined from the boundary condition (2), and the boundary condition (1) is satisfied identically.

According to Equations (2) and (6) we have:

$$
v_{\rho}\left(\rho_{0}\right)=C_{v}\left(\frac{1}{\rho_{0}}-\frac{1}{\rho_{1}}\right)=\frac{V}{\cos \varphi}
$$

from which the dependence for the material moving velocity in the plastic deformation site (PDS) is written as

$$
v_{\rho}=\frac{V}{\cos \varphi} \frac{\frac{1}{\rho}-\frac{1}{\rho_{1}}}{\frac{1}{\rho_{0}}-\frac{1}{\rho_{1}}}
$$

It should be pointed out that realization of dependence (9) violates the assumption made in the form Equation (4) that the plastic flow in the PDS is independent of angle $\varphi$. To avoid this contradiction, we approximate the roller contour with the circumscribed polygon with $\mathrm{N}$ sides, where the number of 
sides $\mathrm{N}$ can be arbitrarily large. In this case, the deformation site can be presented as a set of $\mathrm{N}$ local zones, bounded by angles $\varphi_{0}<\varphi_{1}<\ldots<\varphi_{i}<\ldots<\varphi_{N}$ with radial velocities in each zone.

$$
v_{\rho i}=\frac{V}{\cos \varphi_{i}}\left(\frac{1 / \rho-1 / \rho_{1}}{1 / \rho_{0}\left(\varphi_{i}\right)-1 / \rho_{1}}\right)
$$

In this case, condition (4) for each $i$ zone is not violated, and between adjacent zones $i$ and $i+1$ there is a gap in the peripheral velocity components $\Delta v_{\rho i}=\left|v_{\rho i+1}-v_{\rho i}\right|$, which also does not violate the condition for the existence of a kinematically admissible velocity field, according to which only discontinuities in the normal components of the plastic flow velocity are unacceptable.

From Figure 1 we define the boundary conditions for the PDS geometry.

$$
\begin{aligned}
& \rho_{0}\left(\varphi_{N}\right)=\rho_{1} \\
& \rho_{0}\left(\varphi_{N}\right) \sin \left(\varphi_{N}+\pi\right)+e b=R \\
& \rho_{0}\left(\varphi_{N}\right) \cos \left(\varphi_{N}+\pi\right)=e a \\
& e b+t-\rho_{1} \sin \varphi_{0}=R \\
& e b-\rho_{0}(\varphi) \sin \varphi_{0}=R \sin \alpha_{0} \\
& \left(\rho_{0}(\varphi) \sin \varphi+e b\right)^{2}+\left(\rho_{0}(\varphi) \cos \varphi+e a\right)^{2}=R^{2}
\end{aligned}
$$

From these conditions, the following geometry dimensions can be obtained:

$$
\begin{gathered}
\cos \left(\varphi_{N}+\pi\right)=\frac{e a}{\sqrt{e a^{2}+(R-e b)^{2}}} \\
\varphi_{N}=\arccos \left(\frac{e a}{\sqrt{e a^{2}+(R+e b)^{2}}}\right)-\pi \\
\rho_{1}=\frac{e a}{\cos \left(\varphi_{N}+\pi\right)} \\
\varphi_{0}=\arcsin \left(\frac{e b+t-R}{\rho_{1}}\right) \\
\rho_{0}\left(\varphi_{0}\right)=-1 / 2\left(\left(\sqrt{b^{2}-4 c}\right)-b\right)
\end{gathered}
$$

where

$$
b=2\left(e a \cos \varphi_{0}+e b \sin \varphi_{0}\right), \mathrm{c}=\mathrm{ea}^{2}+e b^{2}-R^{2}
$$

To determine the $e a, e b$ permissible values we use the condition of minimum plastic deformation energy.

Theorem on deformation power upper bound approach method was originally formulated for rigid-plastic materials and predicated that the kinematically admissible velocity field in the PDS minimizes the plastic deformation power. Druker and Providence [22] gave an extended statement of the theorem for the velocity field with discontinuities:

$$
\begin{gathered}
J^{*}=J_{p}+J_{V}+J_{m}-J_{\text {out }} \\
J^{*}=\frac{2}{\sqrt{3}} \int_{W} \sigma_{S} \sqrt{\frac{1}{2} \dot{\varepsilon}_{i j} \dot{\varepsilon}_{i j}} d W+\frac{1}{\sqrt{3}} \int_{S} \sigma_{S}\left|\Delta V_{i}\right| d S+\int_{S_{m}} m \sigma_{s}\left|\Delta V_{i}\right| d S- \\
-\int_{S_{F}} F_{i} V_{i} d S
\end{gathered}
$$

where 
$J_{p}, J_{V}, J_{m}, J_{\text {out }}$ - plastic deformation, sliding along the flow rate discontinuity surfaces, friction forces, and external forces power respectively;

$\dot{\varepsilon}=2 \sqrt{\frac{1}{2} \dot{\varepsilon}_{i j} \dot{\varepsilon}_{i j}}$-shear deformation rate intensity in the deformation site of volume $W$;

$S$-discontinuity surface of velocity peripheral component $\Delta V_{i}$;

$S_{m}$-friction surface (in the roller and medium contact area) with friction coefficient $m$;

$S_{F}$-surface on which external forces $F_{i}$ are active.

According to the extreme principles of solid mechanics, a kinematically admissible velocity field close to the real one minimizes the function of $J^{*}$

$$
J=\min J^{*}
$$

The function, determining the plastic deformation energy, is defined by the dependence

$$
J_{p}=\frac{\sigma_{s}}{\sqrt{3}} \int_{\rho_{0}}^{\rho_{1}} \dot{\varepsilon} \cdot \rho d \rho
$$

where the shear deformation rates intensity is given as

$$
\dot{\varepsilon}=\frac{2}{\sqrt{3}} \sqrt{\dot{\varepsilon}_{\rho \rho}^{2}-\dot{\varepsilon}_{\rho \rho} \dot{\varepsilon}_{\varphi \varphi}+\dot{\varepsilon}_{\varphi \varphi}^{2}+3 / 4 \dot{\varepsilon}_{\rho \varphi}}
$$

and $\sigma_{s}$ is the rigid-plastic body yield strength.

Taking into account Equations (3), (10) and (21):

$$
\dot{\varepsilon}_{i}=2 \dot{\varepsilon}_{\rho \rho i}=-\frac{2 V}{\cos \varphi_{i}} \cdot \frac{1 / \rho^{2}}{1 / \rho_{0}\left(\varphi_{i}\right)-1 / \rho_{1}}
$$

Therefore, using Equation (13), we deriver following from Equation (10):

$$
J_{p}=\sum_{i=1}^{N} J_{p i}=\frac{2}{\sqrt{3}} \sigma_{s} V \sum_{i=1}^{N} \frac{\ln \left(\rho_{1} / \rho_{0}\left(\varphi_{i}\right)\right)}{1 / \rho_{0}\left(\varphi_{i}\right)-1 / \rho_{1}} \cdot \frac{\left(\varphi_{i}-\varphi_{i-1}\right)}{\cos \varphi_{i}}
$$

The friction force power on the roller surface is defined by the following dependence

$$
J_{m}=\int_{S_{m}} m \sigma_{s}\left|\Delta V_{i}\right| d S=m \sigma_{s} \int_{\alpha_{0}}^{\alpha_{1}}\left|V_{\tau}\left(\rho_{0}\right)\right| R d \alpha
$$

where

$$
V_{\tau}\left(\rho_{0}\right)=V_{\rho}\left(\rho_{0}\right) \sin (\alpha-\varphi)=V \frac{\sin (\alpha-\varphi)}{\cos \varphi}=V(\sin \alpha-\cos \alpha \cdot \operatorname{tg} \varphi)
$$

The last integral (24) can be expressed in terms of variables $\alpha, e a, e b$. To do so, we make a substitution

$$
\operatorname{tg} \varphi=\frac{R \sin \alpha-e b}{R \cos \alpha-e a}
$$

Then

$$
J_{m}=m \sigma_{s} V R \int_{\alpha_{0}}^{\alpha_{1}}\left|\sin \alpha-\frac{R \sin \alpha-e b}{R \cos \alpha-e a} \cos \alpha\right| d \alpha
$$


After transformation (26) and integration, we have:

$$
J_{m}=m \sigma_{s} V\left|e a \cdot \ln \left(\frac{R \cos \alpha_{1}-e a}{R \cos \alpha_{0}-e a}\right)+e b \cdot\left(\alpha_{1}-\alpha_{0}\right)\right|
$$

Obviously, a shift due to the difference in the flow velocities along the boundaries will be observed along the boundaries between zones, constrained by angles $\varphi_{0}<\varphi_{1}<\ldots<\varphi_{i}<\ldots<\varphi_{N}$ Therefore

$$
\begin{aligned}
& J_{V}=\sum_{i=1}^{N} J_{V i}=\frac{\sigma_{s}}{\sqrt{3}} \sum_{i=1}^{N} \int_{S}\left|\Delta V_{i}\right| d S \\
& J_{V}=\frac{\sigma_{S}}{\sqrt{3}} \sum_{i=1}^{N} \int_{\rho_{0}\left(\varphi_{i}\right)}^{\rho_{1}}\left|V_{\rho i}-V_{\rho i-1}\right| d \rho= \\
& =\frac{\sigma_{S}}{\sqrt{3}} V \sum_{i=1}^{N}\left(\frac{1}{\cos \varphi_{i}}-\frac{1}{\cos \varphi_{i-1}}\right) \int_{\rho_{0}\left(\varphi_{i}\right)}^{\rho_{1}}\left(\frac{1 / \rho-1 / \rho_{1}}{1 / \rho_{0}\left(\varphi_{i}\right)-1 / \rho_{1}}\right) d \rho= \\
& =\frac{\sigma_{s}}{\sqrt{3}} V \sum_{i=1}^{N}\left(\frac{1}{\cos \varphi_{i}}-\frac{1}{\cos \varphi_{i-1}}\right)\left|\frac{\rho_{1}\left(\ln \left(\rho_{1} / \rho_{0}\left(\varphi_{i}\right)\right)+\rho_{0}\left(\varphi_{i}\right) / \rho_{1}-1\right)}{\rho_{1} / \rho_{0}\left(\varphi_{i}\right)-1}\right|
\end{aligned}
$$

When $N \rightarrow \infty$

$$
\begin{aligned}
& J_{V}=\frac{\sigma_{s}}{\sqrt{3}} V \int_{\varphi_{0}}^{\varphi_{N}} \frac{\partial}{\partial \varphi}\left(\frac{1}{\cos \varphi}\right) d \varphi \int_{\rho_{0}\left(\varphi_{i}\right)}^{\rho_{1}}\left(\frac{1 / \rho-1 / \rho_{1}}{1 / \rho_{0}\left(\varphi_{i}\right)-1 / \rho_{1}}\right) d \rho= \\
& =\frac{\sigma_{s}}{\sqrt{3}} V \int_{\varphi_{0}}^{\varphi_{N}} \frac{d \cos \varphi}{\cos ^{2} \varphi} \int_{\rho_{0}\left(\varphi_{i}\right)}^{\rho_{1}}\left(\frac{1 / \rho-1 / \rho_{1}}{1 / \rho_{0}\left(\varphi_{i}\right)-1 / \rho_{1}}\right) d \rho
\end{aligned}
$$

Making an approximate replacement $\rho_{0}\left(\varphi_{i}\right) \approx \rho_{\text {mid }}=\frac{\rho_{0}+\rho_{1}}{2}$, we obtain

$$
J_{V}=\left(\frac{1}{\cos \varphi_{1}}-\frac{1}{\cos \varphi_{0}}\right)\left|\frac{\rho_{1}\left(\ln \left(\rho_{1} / \rho_{\text {mid }}\right)+\rho_{0} / \rho_{1}-1\right)}{\rho_{1} / \rho_{\text {mid }}-1}\right|
$$

When indenter penetrates the work piece the plastic deformation site is small, so it is reasonable to consider the average volume deformation intensity rates in the form (31).

$$
\dot{\varepsilon}_{\text {mid }}=\frac{J}{\sigma_{s} \cdot V \cdot W}
$$

where

$W=\sum_{i=1}^{N}\left(\rho_{1}^{2}-\rho_{0}\left(\varphi_{i}\right)^{2}\right)\left(\varphi_{i-1}-\varphi_{i}\right)$-plastic deformation zone volume.

Consider the average intender relative downward pressure. According to Equation (19), the total power, spent on plastic deformation and external friction is balanced by external forces applied to the indenter and aligned with the indenter moving velocity vector $V$. Accordingly, for the contact area, turned through angle $\varphi_{i}$ relative to the indenter velocity vector, the indenter downward pressure is defined as the projection of the normal component of the resulting external stress $p_{s}$ on the pressing down direction (Figure 4).

$$
p_{i}=\frac{J_{p i}+J_{V i}+J_{m i}}{V} \cos \left(\varphi_{i}\right) \sin \left(\varphi_{i}\right)
$$

Then, considering the dependencies Equations (23), (27), (28), and (32), the downward pressure on the interface $p$ is determined by equation: 


$$
\begin{aligned}
& p=\frac{\sigma_{s}}{R\left(\alpha_{0}-\alpha_{N}\right)} \sum_{i=1}^{N} \frac{2}{\sqrt{3}} \frac{\ln \left(\rho_{1} / \rho_{0}\left(\varphi_{i}\right)\right)}{1 / \rho_{0}\left(\varphi_{i}\right)-1 / \rho_{1}}\left(\varphi_{i-1}-\varphi_{i}\right) \sin \left(\varphi_{i}\right)+ \\
& \frac{1}{\sqrt{3}}\left(1-\frac{\cos \left(\varphi_{i}\right)}{\cos \varphi_{i-1}}\right)\left|\frac{\rho_{1}\left(\ln \left(\rho_{1} / \rho_{0}\left(\varphi_{i}\right)\right)+\rho_{0}\left(\varphi_{i}\right) / \rho_{1}-1\right)}{\rho_{1} / \rho_{0}\left(\varphi_{i}\right)-1}\right|\left(\varphi_{i}\right) \sin + \\
& +\frac{m}{2}\left|e a \cdot \ln \left(\frac{R \cos \alpha_{i}-e a}{R \cos \alpha_{i-1}-e a}\right)+e b \cdot\left(\alpha_{i}-\alpha_{i-1}\right)\right| \sin \left(2 \varphi_{i}\right)
\end{aligned}
$$

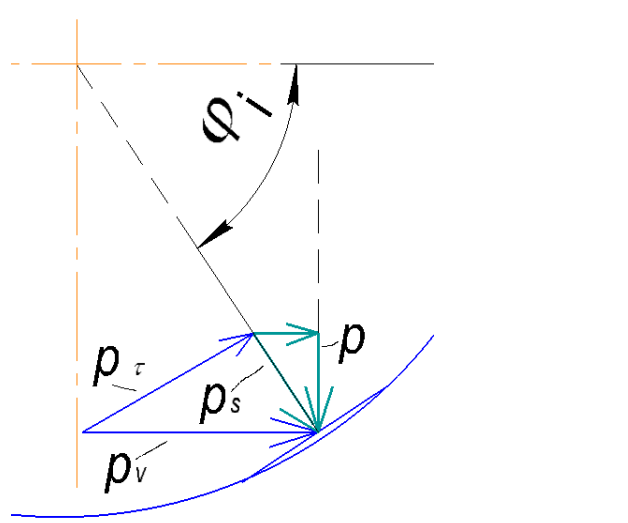

Figure 4. Determining indenter pressing down stress p.

Dependences (33) are obtained using the kinematically admissible velocity field and satisfy the boundary conditions (1) and (2). The unknown parameters $e a, e b$, determining the plastic deformation site geometry and coordinates, can be determined from the widened theorem on plastic deformation power upper boundary in the form Equation (19). Thus, the problem of determining the WB kinematically admissible velocity field is reduced to the optimization problem of two variables in the formulation:

$$
\operatorname{argmin} J(\operatorname{var}) \in\left\{\operatorname{var}=(e a, e b) \mid \forall p_{0}=\left(e a_{0}, e b_{0}\right): J(\operatorname{var}) \leq J\left(\operatorname{var}_{0}\right)\right\}
$$

The solutions for relative pressure on indenter $\sigma=p$ p/yield stress, where $p$ is determined by Equation (33, are presented in Figure 5. The penetration depth $t=0.004-0.01 \mathrm{~mm}$ in in Figure 5 corresponds to a surface roughness decrease in $\mathrm{mm}$

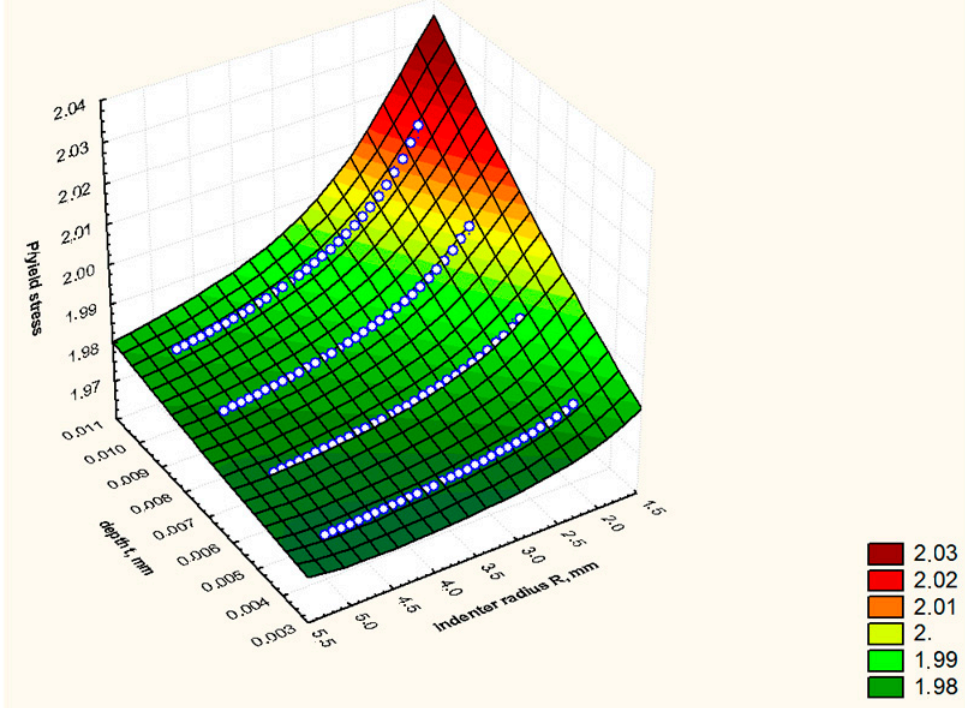

Figure 5. Theoretical dependence of the relative pressure on indenter $\sigma=p / y i e l d$ stress from the intender penetration depth $\mathrm{t}$ and its radius $\mathrm{R}$. 
To substantiate the theoretical model reliability in Equations (1)-(34), a numerical experiment on wide burnishing was carried out using the finite elements method.

To justify the possibility of a kinematically admissible velocity field, as shown in Figure 3, finite elements analysis was performed in Deform 2D, while fulfilling boundary conditions (1) and (2). Figure 6 shows the field of material displacement velocities during burnishing, obtained based on the results of the finite elements analysis (FEA). To ensure comparability with the theoretical analysis results, hereafter the rigid plastic material (yield stress $=1 \mathrm{MPa}$ ) was considered in FEA. Minimum mesh size of the finite elements mesh was equal to $0.0035 \mathrm{~mm}$.

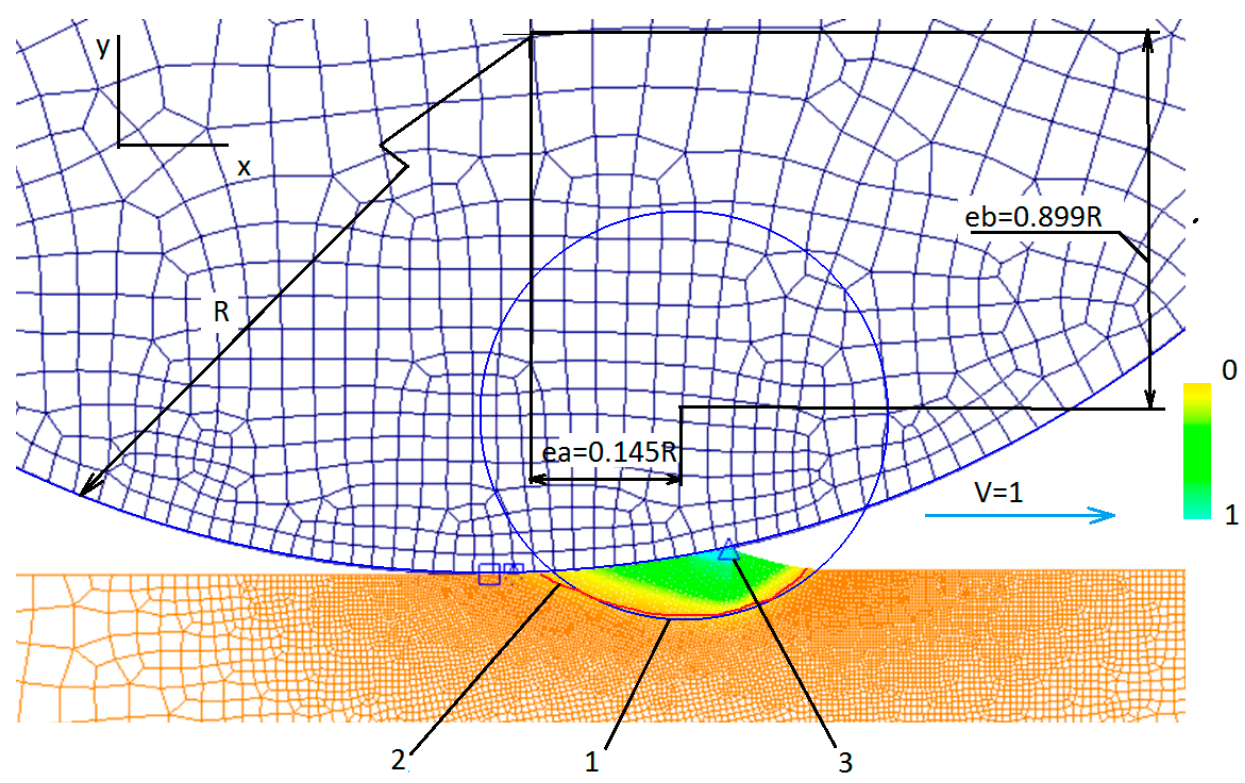

Figure 6. Material displacement area boundaries when burnishing $(t=0.008 \mathrm{~mm}, \mathrm{R}=2 \mathrm{~mm})$.

1-approximation of the displacement area boundary by a circle according to the theoretical model; 2-boundary for area, calculated by the finite elements method; 3-field of material moving velocity

Accepted indenter movement velocity $-1 \mathrm{~mm} / \mathrm{s}$. The analysis shows that the material displacement area boundary can be inscribed in a circle with center, located at a distance eb $=0.899 \mathrm{R}$, ea $=0.145 \mathrm{R}$, where $\mathrm{R}$ is the indenter radius. Found ratios for eb and ea were verified for $\mathrm{R}$ values from 0 to 5 and $\mathrm{t}$ $=0.004 \ldots 0.02$.

Some of the finite element analysis results for various depths of indenter penetration are shown in Figures 7 and 8. Average relative pressure is determined numerically as

$$
\sigma y=\frac{\sum_{i=1, N} Y_{i} S_{i}}{\sum_{i=1, N} S_{i}}
$$

where $Y_{i}$ (Stress $Y$ in Figures 7 and 8) is the vertical pressure component for the i-finite element (group of elements) on a contact arc with length of $S_{i}$. 


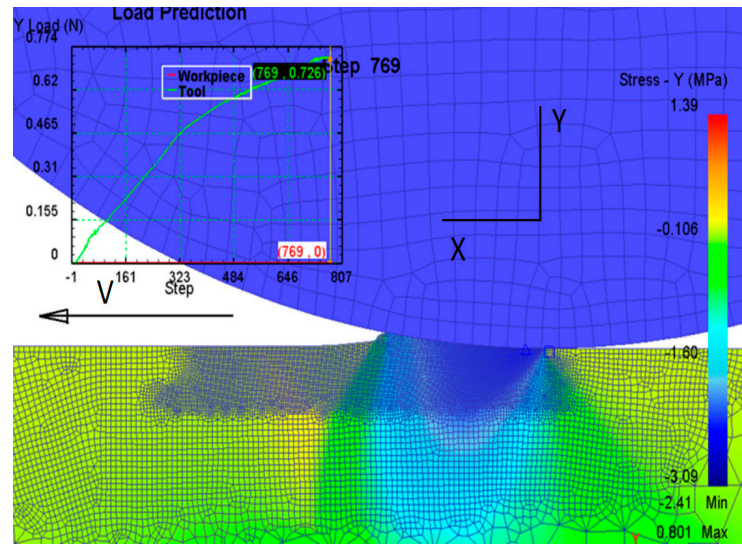

(a)

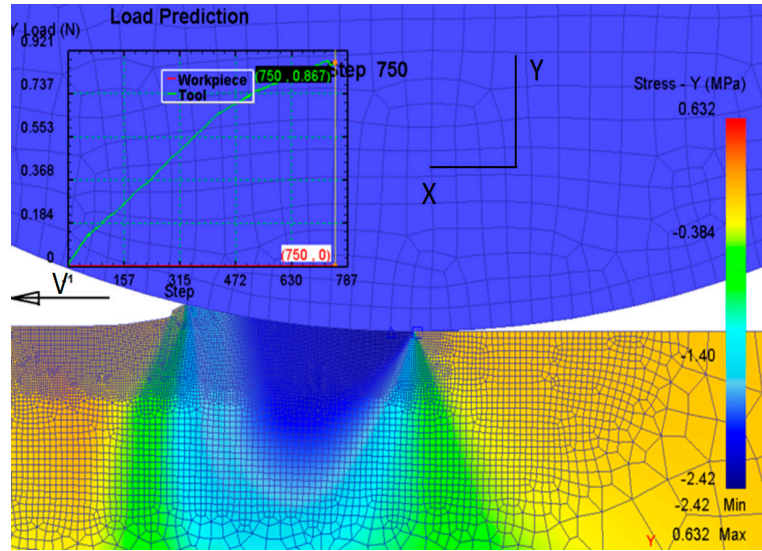

(b)

Figure 7. Dependence of the relative pressure by Equation (35) (yield stress $=1 \mathrm{MPa}$ ) during finite elements simulation of wide burnishing by indenter $R=2 \mathrm{~mm}$ at $(\mathbf{a}) \mathrm{t}=0.004 \mathrm{~mm}$; $(\mathbf{b}) \mathrm{t}=0.008 \mathrm{~mm}$.

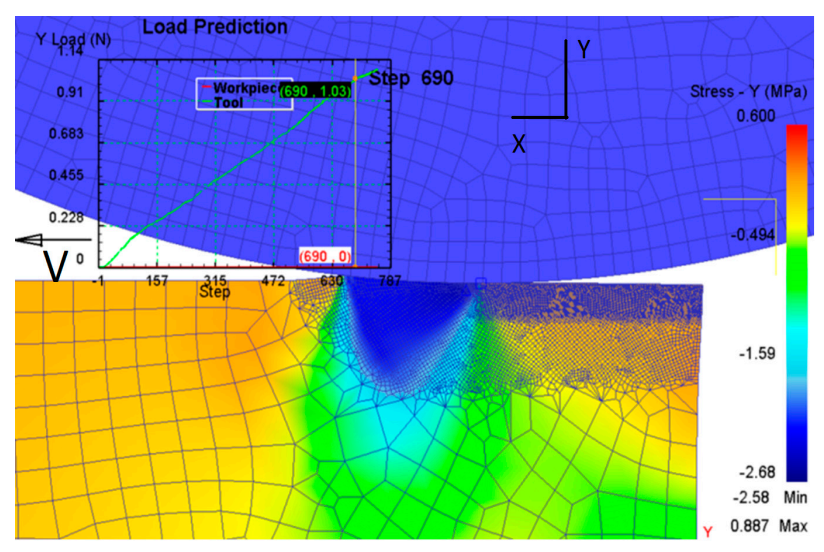

(a)

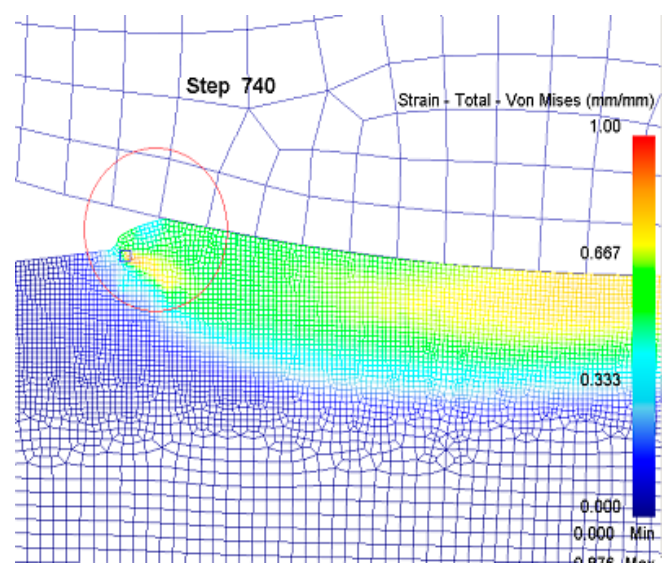

(b)

Figure 8. (a) Dependence of the relative pressure by Equation (35) (yield stress = $1 \mathrm{MPa}$ ) during finite elements simulation of wide burnishing by indenter $\mathrm{R}=4 \mathrm{~mm}$ at $\mathrm{t}=0.009 \mathrm{~mm}$; (b) bulge forming in front of the indenter $(\mathrm{R}=2 \mathrm{~mm}, \mathrm{t}=0.008 \mathrm{~mm})$.

Table 1 shows the results of a comparative analysis of the average relative pressure calculation by the numerical finite elements method in Deform 2D and by analytical Equations (1)-(34).

Table 1. Comparative analysis of determining the average relative pressure by numerical and analytical methods.

\begin{tabular}{cccccc}
\hline $\begin{array}{c}\text { Depth t, } \\
\text { mm }\end{array}$ & $\begin{array}{c}\text { Indenter } \\
\text { Radius R, } \\
\text { mm }\end{array}$ & $\mathbf{R / t}$ & $\begin{array}{c}\text { Average Pressure by Equation } \\
\text { (33) Analytical Calculation } \\
\text { (Yield Stress = 1 MPa) }\end{array}$ & $\begin{array}{c}\text { Average Pressure by } \\
\text { Equation (35) Finite } \\
\text { Elements Calculation } \\
\text { (Yield Stress = 1 MPa) }\end{array}$ & $\begin{array}{c}\text { Relative } \\
\text { Error }\end{array}$ \\
\hline 0.004 & 2 & 500 & 1.981 & 1.85 & $7.1 \%$ \\
0.008 & 2 & 500 & 2.002 & 1.99 & $0.6 \%$ \\
0.004 & 4 & 1250 & 1.973 & 1.81 & $9.0 \%$ \\
0.008 & 4 & 1250 & 1.981 & 1.89 & $4.8 \%$ \\
0.02 & 4 & 200 & 2.016 & 2.1 & $-4.0 \%$ \\
\hline
\end{tabular}

From the data provided in Table 1, it follows that the analytical method gives a top boundary estimate of the average indenter penetration pressure. For small penetration depths, the difference increases up to $9 \%$. As the indenter penetration depth increases, a bulge forms in front of the indenter 
during burnishing (Figure 8b), which is taken into account when calculating the average penetration pressure in the finite elements analysis and is not taken into account in the analytical model. This leads to negative error values of the analytical model (row 5 of Table 1 ) at large penetration depths. It should be noted that in practice significant burnishing depths are not used.

\section{Experimental Procedure}

AISI 1040 steel bars with a tensile strength of $590 \mathrm{MPa}$ (quenching, tempering) and casts from high-strength cast iron 75-50-03 with a yield strength of $750 \mathrm{MPa}$ were subjected to wide burnishing according to scheme (Figures 1 and 2). Figures 9 and 10 demonstrate variation of the surface roughness $\mathrm{Ra}, \mu \mathrm{m}$ (arithmetical mean determined from deviations from the center line) depending on the force per unit length of the indenter. The initial roughness of the AISI 1040 steel bars was equal to $\mathrm{Ra}=0.5 \mu \mathrm{m}$ and for cast iron it was equal to $\mathrm{Ra}=0.86 \mu \mathrm{m}$. The roughness of the treated surface was measured after each sequence of 4,6 and 10 passes. Fitted curves for the experimental points were constructed using the distance-weighted least squares method. The resulting curves have a clearly defined minimum of roughness value.

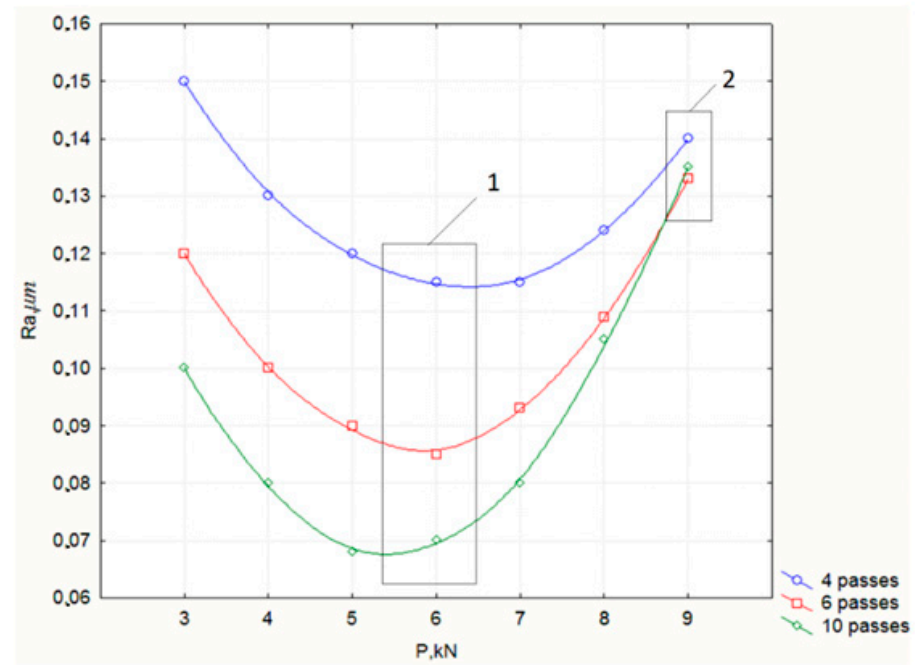

Figure 9. The surface roughness variation depending on the WB indenter pressure for AISI 1040 work piece.

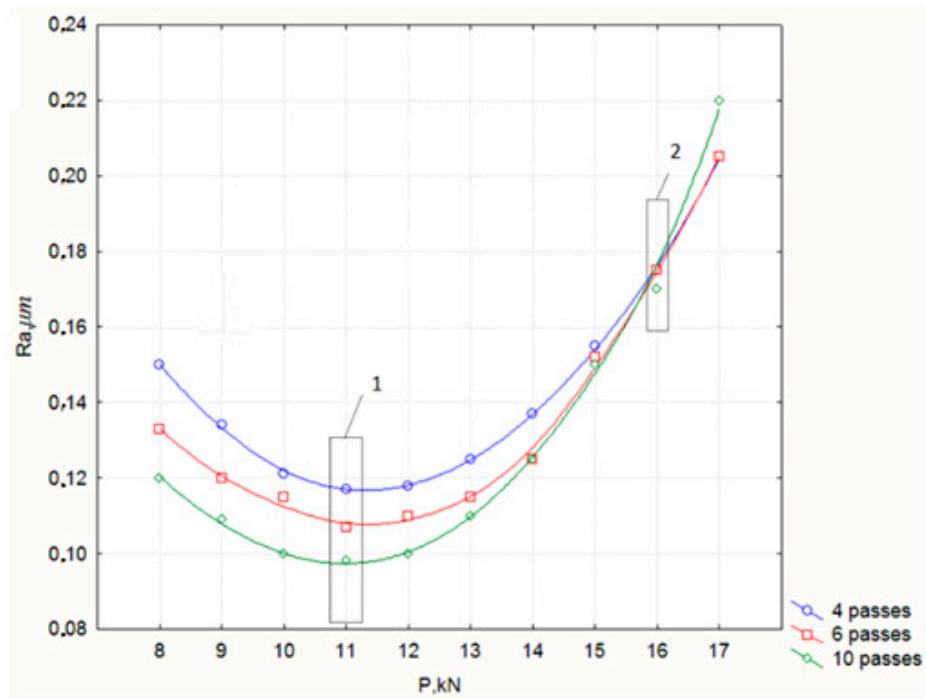

Figure 10. The surface roughness variation depending on the WB indenter pressure for 75-50-03 cast iron work piece. 


\section{Discussion}

With a properly selected burnishing mode, the protruding irregularities of the surface microrelief are deformed with increasing their cross section in the plane, formed by the indenter pressure force vectors and the burnishing speed. Thus, microroughness peaks are decreased and valleys are filled. Usually burnishing is carried out in several passes, since with an increased indenter pressure, material squeezes out in front of the indenter and a bulge forms, contributing to an undesired increase in roughness. As the microroughness valleys fill up, an increase in the number of passes with a fixed amount of pressure also does not contribute to a further decrease in roughness (Figures 9 and 10). When analyzing the graphs in Figures 9 and 10, two characteristic areas can be distinguished-area 1 of the minimum roughness values, corresponding to the region of optimal indenter pressure values. Area 2 is characterized by constant roughness with a different number of burnishing passes. This means that starting from the first passes, the indenter-surface contact area increases to the maximum theoretical value (see Equation (37)) due to deforming all microroughnesses. Corresponding to this area, pressure value is the boundary, exceeding of which leads to the indenter being pressed into solid metal.

The specific pressure that reduce surface roughness is determined by the following ratio:

$$
\sigma y_{p}=\frac{F \cdot n}{\sigma_{t} \cdot L}
$$

where $F$ - the indenter pressure related to its length;

$$
L=\sqrt{2 R\left(R a_{0}-R a_{1}\right)-\left(R a_{0}-R a_{1}\right)^{2}}
$$

— the theoretical contact line in the indenter cross section, $R$ is the indenter radius, $R a_{0}, R a_{1}$ 一the initial and resulting surface roughness, respectively; $n$ — the number of burnishing passes; $\sigma_{t}$-yield stress.

Results of the burnishing experiments analysis are summarized in Table 2.

\begin{tabular}{|c|c|c|c|c|c|}
\hline Material & $F, \mathrm{kN} / \mathrm{mm}$ & $R a_{0}, \mu \mathrm{m}$ & $R a_{1}, \mu \mathrm{m}$ & $\mathbf{n}$ & $\sigma y_{p}$ \\
\hline $\begin{array}{c}\text { AISI } 1040 \\
\text { area 1, Figure } 9\end{array}$ & 6 & 0.5 & 0.115 & 4 & 1.55 \\
\hline $\begin{array}{c}\text { AISI } 1040 \\
\text { area 2, Figure } 9\end{array}$ & 9 & 0.5 & 0.12 & 4 & 1.57 \\
\hline $\begin{array}{l}\text { cast iron } 75-50-03 \\
\text { area } 1 \text {, Figure } 10\end{array}$ & 11 & 0.86 & 0.11 & 4 & 1.56 \\
\hline $\begin{array}{l}\text { cast iron } 75-50-03 \\
\text { area } 2 \text {, Figure } 10\end{array}$ & 16 & 0.86 & 0.17 & 4 & 1.62 \\
\hline
\end{tabular}

Table 2. Results of the burnishing experiments analysis (Figures 9 and 10).

The optimal indenter pressure initiates a plastic flow, which ensures the filling of valleys with a minimum number of passes. Analysis of roughness vs indenter pressure dependences curves shows that the bulk displacement of the microroughness material (up to $86 \%$ ) falls in the first four passes. On the 10th pass, as shown by the curves of the roughness growth after exceeding the indenter pressures optimal values, roughness dramatically increasing is observed, which is explained by forming a significant bulge in front of the indenter during its movement.

Conditionally taking the roughness values difference before and after burnishing as the indenter penetration depth, $t \approx R a_{0}-R a_{1}$, you can compare the pressure on the indenter data from analytical and numerical analysis (Table 1, columns 4 and 5) with the experimental results (Table 2, column 6). The comparison shows that: 
(1) The pressure on indenter, found by the analytical method, is up to $9 \%$ greater than the pressure determined by the finite elements method, which is explained by implementing the upper bound approach in the analytical method;

(2) The pressure on indenter, found experimentally using Equation (37), is on average 20\% less than the pressure calculated by the finite elements method.

A significant $20 \%$ difference between the numerical and experimental data is explained by the fact that real indenter contact surface, determined by the contacting surface micro relief, is always less than the calculated theoretical L in Equation (37).

\section{Conclusions}

As a result of the performed studies, analytical dependences and simulation results of numeric FE-method were obtained for the energy-force parameters of the wide burnishing, such as the specific indenter downward pressure and the average deformation rate in the plastic deformation site, forming when moving indenter penetrates the work piece surface. The analytical upper bound approach method demonstrated a convergence of up to $9 \%$ with FEM when determining the indenter penetration pressure. Experiments on choosing the optimal burnishing parameters made it possible to determine the upper limit of the indenter penetration pressure, which values are less than 1.6 of the material yield stress under indenter four passes when burnishing. Specific values of the penetration forces optimal parameters depend on the surface micro relief and should be specified experimentally.

Author Contributions: Conceptualization, methodology, supervision, I.B.; formal analysis, writing-original draft preparation, A.K.; investigation, writing - review and editing, N.B.; data curation, visualization, A.D. All authors have read and agreed to the published version of the manuscript.

Funding: This research was funded by the grant of Russian Science Foundation (project NO 19-19-00171).

Acknowledgments: The authors would like to thank Alexander Sverchkov, director of engine production of AVTOVAZ JSC for many years of work support.

Conflicts of Interest: The authors declare no conflict of interest.

\section{References}

1. Yen, Y.C.; Sartkulvanich, P.; Altan, T. Finite Element Modeling of Roller Burnishing Process. CIRP Ann. 2005, 54, 237-240. [CrossRef]

2. Prevey, P.S.; Ravindranath, R.A.; Shepard, M.; Gabb, T. Case Studies of Fatigue Life Improvement Using Low Plasticity Burnishing in Gas Turbine Engine Applications. Trans. ASME 2006, 128, 866-872. [CrossRef]

3. Hassan, A.M.; Maqableh, A.M. The Effects of Initial Burnishing Parameters on Non Ferrous Components. J. Mater. Process. Technol. 2000, 102, 115-121. [CrossRef]

4. El-Axir, M.H.; EI-CKhabeery, M.M. Influence of Orthogonal Burnishing Parameters on Surface Characteristics for Various 4. Materials. J. Mater. Process. Technol. 2003, 132, 82-89. [CrossRef]

5. Nemat, M.; Lyons, A.C. An Investigation of the Surface Topography of Ball Burnished Mild Steel and Aluminum. Int. J. Adv. Manuf. Technol. 2000, 16, 469-473.

6. Shiou, F.J.; Chen, C.H. Determination of Optimal Ball Burnishing Parameters for Plastic Injection Moulding Steel. Int. J. Adv. Manuf. Technol. 2003, 3, 177-185.

7. Roettger, K. Walzen Hartgedrehter Oberflaechen. Ph.D. Thesis, RWTH Aachen, North Rhine-Westphalia, Gremany, 2002.

8. Howell, M.; Hahn, G.T.; Rubin, C.A.; McDowell, D.L. Finite Element Analysis of Rolling Contact for Nonlinear Kinematic Hardening Bearing Steel. J. Tribol. 1995, 117, 729-736. [CrossRef]

9. Shiratori, M.; Ito, M.; Hashimoto, M. Elastic-Plastic Analysis of Rolling Contact for Surface Hardened Steel. Trans. Jpn. Soc. Mech. Eng. A. 1995, 61, 1064-1069. [CrossRef]

10. Marshall, E.A. Rolling Contact with Plastic Deformation. J. Mech. Phys. Solids 1968, 16, 243-254. [CrossRef]

11. Collins, I.F. A Simplified Analysis of the Rolling of Cylinder on a Rigid/Perfectly Plastic Half-Space. Int. J. Mech. Sci. 1972, 14, 1-14. [CrossRef] 
12. Nepershin, R.I. The Rolling and Slipping of a Cylinder along the Boundary of an Ideally Plastic Half-Space. J. Appl. Math. Mech. 2003, 67, 293-301. [CrossRef]

13. Nepershin, R.I. Plastic Deformation of Surface Layer during Rigid Cylinder Rolling and Sliding. J. Frict. Wear 2013, 34, 204-207. [CrossRef]

14. Nepershin, R.I. Surface Plastic Deformation by Sliding Elliptical Cylinder. J. Mater. Sci. Chem. Eng. 2015, 3, $1-7$.

15. Pietrzyk, M.; Madej, L.; Rauch, L.; Szeliga, D. Computational Materials Engineering. In Achieving High Accuracy and Efficiency in Metals Processing Simulations; Elsevier: Amsterdam, The Netherlands, 2015; 381p.

16. Prager, W. The Theory of Plasticity: A Survey of Recent Achievements. Proc. Inst. Mech. Eng. 1955, 169, 41-57. [CrossRef]

17. Kudo, H. Upper-bound Approach to Metal Forming Processes-to Date and in the Future. In Metal Forming and Impact Mechanics; Reid, S.R., Ed.; Pergamon Press: Oxford, UK, 1985; pp. 19-45.

18. Johnson, W.; Mellor, P.B. Engineering Plasticity; Ellis Horwood Ltd.: Chichester, UK, 1983; 646p.

19. Avitzur, B. Metal Forming Processes and Analysis; McGraw-Hill Book Co.: New York, NY, USA, 1968; pp. 102-111.

20. Grechnikov, F.; Khaimovich, A. The study of plastic deformation at high deformation rates in upset forging of cylinders. Key Eng. Mater. 2016, 684, 74-79. [CrossRef]

21. Abulkhanov, S.R.; Skuratov, D.L.; Khaimovich, A.I.; Shvetcov, A.N. Analytical approach to determining of parameters which characterize surface layer quality of the parts hardened by a traveling diamond sphere. Int. J. Eng. Technol. 2014, 6, 2191-2200.

22. Druker, D.C.; Providence, R.I. Coulomb friction. J. Appl. Mech. 1954, 21, 71-74.

(C) 2019 by the authors. Licensee MDPI, Basel, Switzerland. This article is an open access article distributed under the terms and conditions of the Creative Commons Attribution (CC BY) license (http://creativecommons.org/licenses/by/4.0/). 